\title{
Henamientas terapéuticas para los pacientes con enfermedad hepática grasa no alcohólica
}

\author{
Therapeutic tools for patient with nonalcoholic steatohepatitis
}

\section{Objetivo}

Revisar la evidencia disponible sobre la utilidad de diferentes estrategias terapéuticas (farmacológicas y no farmacológicas) para el tratamiento de la enfermedad hepática grasa no alcohólica (EHGNA).

\section{Fuente y selección de datos}

Se revisaron hasta enero de 2010 MEDLINE, Ovid MEDLINE InProcess, Cochrane Database of Systematic Reviews, Excerpta Medica Database, Pubmed y clinicaltrials.gov., y resúmenes de los trabajos presentados en las reuniones científicas hepatológicas y gastroenterológicas más importantes de Estados Unidos y Europa. Fueron incluidos aquellos ensayos clínicos aleatorizados que hubieran comparado una o más estrategias para el tratamiento de la EHGNA en pacientes diagnosticados por técnicas de imágenes o biopsia. El objetivo primario de los tratamientos fue la respuesta histológica (grado de esteatosis, inflamación o fibrosis). Cuando no se contó con biopsia se evaluó la respuesta bioquímica o de las imágenes (ecografía, tomografía o resonancia/espectroscopía)

\section{Resultados principales}

Fueron incluidos 49 ensayos. Más de la mitad de los estudios no había utilizado biopsia hepática para evaluar los resultados, no superando los dos años el período de intervención/seguimiento. Un descenso de más de un $5 \%$ del peso con dieta, ejercicio y/o drogas (Orlistat) se asociaría a una mejoría de la histología, logrando sólo la mitad de los pacientes descenso adecuado, menos aún su mantenimiento. Diez trabajos utilizaron pioglitazona o rosiglotazona -evaluadas en el contexto de planes dietéticos y de ejercicio de distinta intensidad, contra placebo o metformina o combinándolas con esta última y losartán-, y reportando la mayoría resultados histológicos. En líneas generales las "glitazonas" (principalmente pioglitazona) se asociaron a una mejoría de la esteatosis y la inflamación, pero no de la fibrosis.

Ocho trabajos evaluaron la metformina, la mayoría reportando resultados histológicos y en el mismo contexto de las drogas descriptas previamente. Si bien varios de los estudios demostraron algún grado de mejoría histológica, el número de pacientes incluidos fue substancialmente menor que en los estudios con glitazonas.

También fueron evaluados ácidos grasos poliinsaturados, gemfibrozil, fenofibrato, simvastatina, atorvastatina, probucol, vitaminas C y $\mathrm{E}$, betaína, $\mathrm{N}$-acetilcisteína, telmisartán, valsartán, ácido ursodesoxicólico, pentoxifilina, rimonabant y L-carnitina; sin resultados concluyentes

\section{Conclusiones}

Hasta que se disponga estudios de mayor poder estadístico, mayor número de pacientes y seguimientos más prolongados no existe la posibilidad de establecer una recomendación para el tratamiento de la EHGNA.

Palabras claves: enfermedad hepática, esteatosis, esteatohepatitis, hígado graso, hígado.

Keywords: hepatic disease, esteatosis, steatohepatitis, fat liver.

Fuente de financiamiento: Piedmont Region Funds Interministeriale per la Programmazione Economica 2008

\section{Comentario}

La Enfermedad Hepática Grasa No Alcohólica (EGHNA) es el motivo más frecuente de consulta en hepatología. Su espectro incluye a la esteatosis simple (ES) a la esteatohepatitis no alcohólica (EHNA) y a su eventual evolución a la cirrosis. Los pacientes con ES suelen tener una evolución favorable, mientras que los pacientes con EHNA pueden evoluciona a la cirrosis'.

El principal problema de los estudios que evaluaron los tratamientos para la EHGNA/EHNA es que se trata de una enfermedad no siempre progresiva, y que cuando evoluciona en forma desfavorable lo hace muy lentamente. Por lo tanto, para contar con el poder* suficiente como para detectar diferencias pronósticas que sean clínica y estadísticamente significativas y que puedan ser atribuibles a alguna intervención (farmacológica o no farmacológica) harían falta estudios de seguimiento prolongado (décadas) y que no pudieron ser identificados por los revisores. Por otro lado, dada la dificultad de documentar resultados como mortalidad, incidencia de cirrosis y calidad de vida en el largo plazo, solemos conformarnos con los resultados de la mejoría histológica. Sin embargo -y además de que la biopsia puede sobre o subestimar la magnitud del daño histológico- la regresión de la esteatosis, la inflamación y especialmente de la fibrosis (si es que esto es posible) también son procesos lentos y que requieren mayores períodos de seguimiento que los que informan las investigaciones identificadas.

Consideramos importante identificar a los pacientes en riesgo de evolucionar a cirrosis para intensificar el control del síndrome metabólico y controlar otras posibles variables como el consumo de alcohol y la prevención y/o tratamiento de las hepatitis virales. La biopsia hepática es el único método diagnóstico con el que contamos para estratificar el espectro de la EHGNA, pero no existe acuerdo de cuándo ni a qué pacientes debería solicitarse. El rol de la Elastografía Hepática (Fibroscan) aún no ha sido determinado, pero podría ser una herramienta valiosa por ser un método no invasivo.

\section{Conclusiones del Comentador}

Dado que el pronóstico de los pacientes con EHGNA se relaciona más con los factores de riesgo cardiovasculares (FRV) que con la propia enfermedad hepática, el abordaje de los pacientes con diagnóstico de EHGNA/EHNA debería ir en paralelo al manejo de aquellos (alimentación saludable, ejercicio, manejo de la insulinoresistencia-diabetes y dislipemia) no existiendo evidencia actualmente para recomendar otras intervenciones.

Sebastián Marciano [ SSección de Hepatología, Hospital Italiano de Buenos Aires. sebastian.marciano@hospitalitaliano.org.ar ]

Ver glosario* 2010. Comentado de: Musso G y col. A meta-analysis of randomized trials for the treatment of nonalcoholic fatty liver disease. Hepatology 2010 Jul; 52 (1): 79-104.

Referencias

1. Sheth S y col. Natural history and treatment of nonalcoholic steatohepatitis. En Kaplan M y Bonis P editors. UpToDate 2010.

Disponible en: http://www.uptodate.com/online/content/topic.do?topicKey=hep dis/21864\&selectedTitle=2\%7E38\&source=search result (último acceso 25/10/10). 